\title{
CHANCE COINCIDENCES AND THE SO-CALLED REDSHIFT SYSTEMS IN THE ABSORPTION SPECTRUM OF PKS O237-23
}

\author{
Y. P. VARSHNI \\ Department of Physics, University of Ottawa, Canada
}

(Received 19 March, 1979)

\begin{abstract}
It is shown that the number of redshift systems based on C IV doublets, proposed by Boroson et al. (1978) in the absorption spectrum of the quasar PKS 0237-23, is insignificantly different from that that would be expected from chance coincidences. Consequently, these systems and their $z$-values appear to be devoid of any physical significance.
\end{abstract}

\section{Introduction}

The object PKS 0237-23 was identified as a quasar by Arp et al. (1967) who also discovered that there are many lines in its absorption spectrum. Subsequent investigations on its absorption spectrum were those of Greenstein and Schmidt (1967), Burbidge et al. (1968) and Boksenberg and Sargent (1975).

Recently, Boroson et al. (1978, hereafter BSBC) have reported on the absorption spectrum of PKS 0237-23. Six observations made over 3 years were combined to yield wavelengths and equivalent widths of 193 absorption lines in the spectral region $\lambda \lambda 3715-4290$. These authors claim that many of these absorption lines are due to $\mathrm{C}$ IV $\lambda \lambda 1548.20,1550.77$ at various redshifts. A total of 45 redshifts have been claimed.

We have proposed a theory of quasars (Varshni, 1973, 1974a, 1975a, 1977a, b, 1978, 1979; Varshni and Lam, 1976), based on sound physical principles, which does not need the artificial assumption of redshifts and provides satisfactory explanations of the various phenomena associated with quasars. All the observational evidence on quasars either supports our theory, or is consistent with it (Varshni, 1979). Clearly, the claims of BSBC require examination.

\section{The 'Redshift' Systems of BSBC}

BSBC carried out their search for redshifted C IV lines as follows: "Line pairs identified as $C_{\text {IV }}$ doublets were required to have the correct separation to \pm 0.3 ångstroms. The ratio of the equivalent widths $W(\lambda 1548): W(\lambda 1551)$ should lie between 2 and 1. This requirement was made with appropriate account taken of the accuracy of the equivalent widths. Occasional exceptions were made to this constraint if one of the lines was obviously an unresolved blend." Forty-five 


\section{TABLE I}

'Redshifted' C IV doublets in PKS 0237-23

\begin{tabular}{|c|c|c|c|c|c|c|c|}
\hline \multirow[b]{2}{*}{$\begin{array}{l}\text { System } \\
\text { number }\end{array}$} & \multirow[b]{2}{*}{$z$} & \multicolumn{2}{|c|}{$\mathrm{C}$ IV $\lambda 1548.20$} & \multicolumn{2}{|c|}{ C IV $\lambda 1550.77$} & \multirow[b]{2}{*}{$\mathrm{R}(\mathrm{EW})$} & \multirow[b]{2}{*}{$\begin{array}{l}\text { Credibility } \\
\text { rating }\end{array}$} \\
\hline & & $\lambda(\AA)$ & $\begin{array}{l}\text { Equivalent } \\
\text { width }(\AA)\end{array}$ & $\lambda(\AA)$ & $\begin{array}{l}\text { Equivalent } \\
\text { width }(\AA)\end{array}$ & & \\
\hline 1 & 1.4007 & 3716.9 & 0.6 & 3722.8 & 0.9 & 0.67 & PO \\
\hline 2 & 1.4174 & 3742.6 & 0.3 & 3748.8 & 0.2 & 1.50 & PO \\
\hline 3 & 1.4575 & 3804.7 & 0.5 & 3810.9 & 0.3 & 1.67 & PO \\
\hline $3 a$ & 1.4794 & 3838.5 & 1.2 & 3845.2 & 0.1 & 12.00 & \\
\hline 4 & 1.5023 & 3874.2 & 0.7 & 3880.3 & 0.7 & 1.00 & $\mathrm{C}$ \\
\hline $4 a$ & 1.5063 & 3880.3 & 0.7 & 3886.7 & 1.9 & 0.37 & \\
\hline 5 & 1.5144 & 3892.8 & 0.8 & 3899.3 & 0.3 & 2.67 & PR \\
\hline 6 & 1.5153 & 3894.1 & 0.4 & 3900.7 & 0.3 & 1.33 & PR \\
\hline $6 a$ & 1.5185 & 3899.3 & 0.3 & 3905.5 & 0.9 & 0.33 & \\
\hline 7 & 1.5260 & 3910.8 & 0.2 & 3917.1 & 0.2 & 1.00 & PO \\
\hline 8 & 1.5348 & 3924.6 & 0.3 & 3930.8 & 0.1 & 3.00 & PR \\
\hline 9 & 1.5389 & 3930.8 & 0.1 & 3937.2 & 0.1 & 1.00 & PO \\
\hline $9 a$ & 1.5517 & 3950.6 & 1.0 & 3957.0 & 0.1 & 10.00 & \\
\hline 10 & 1.5546 & 3954.9 & 0.1 & 3961.7 & 0.2 & 0.50 & PO \\
\hline 11 & 1.5559 & 3957.0 & 0.1 & 3963.7 & 0.2 & 0.50 & PR \\
\hline 12 & 1.5603 & 3963.7 & 0.2 & 3970.6 & 0.1 & 2.00 & PR \\
\hline $12 \mathrm{a}$ & 1.5787 & 3992.3 & 0.1 & 3999.0 & 0.1 & 1.00 & \\
\hline 13 & 1.5950 & 4017.6 & 0.7 & 4024.3 & 0.3 & 2.33 & C \\
\hline $13 a$ & 1.5956 & 4018.6 & 0.4 & 4025.1 & 1.2 & 0.33 & \\
\hline 14 & 1.5964 & 4019.8 & 1.2 & 4026.3 & 0.8 & 1.50 & C \\
\hline 15 & 1.6102 & 4041.0 & 0.6 & 4047.8 & 0.4 & 1.50 & C \\
\hline 16 & 1.6146 & 4047.8 & 0.4 & 4054.8 & 0.1 & 4.00 & PO \\
\hline 17 & 1.6164 & 4050.6 & 0.1 & 4057.6 & 0.1 & 1.00 & PO \\
\hline 18 & 1.6209 & 4057.6 & 0.1 & 4064.4 & 0.1 & 1.00 & PO \\
\hline 19 & 1.6311 & 4073.4 & 0.1 & 4080.4 & 0.3 & 0.33 & PO \\
\hline 20 & 1.6356 & 4080.4 & 0.3 & 4087.2 & 0.2 & 1.50 & PR \\
\hline 21 & 1.6365 & 4081.7 & 0.2 & 4088.7 & 0.1 & 2.00 & PR \\
\hline 22 & 1.6399 & 4087.2 & 0.2 & 4093.9 & 0.1 & 2.00 & PR \\
\hline 23 & 1.6410 & 4088.7 & 0.1 & 4095.7 & 0.2 & 0.50 & PO \\
\hline 24 & 1.6429 & 4091.7 & 0.1 & 4098.5 & 0.1 & 1.00 & PO \\
\hline 25 & 1.6443 & 4093.9 & 0.1 & 4100.7 & 0.1 & 1.00 & PO \\
\hline 26 & 1.6512 & 4104.5 & 0.1 & 4111.4 & 0.9 & 0.11 & PO \\
\hline 27 & 1.6556 & 4111.4 & 0.9 & 4118.2 & 0.7 & 1.29 & $\mathrm{C}$ \\
\hline 28 & 1.6568 & 4113.2 & 0.9 & 4120.2 & 1.1 & 0.82 & C \\
\hline 29 & 1.6581 & 4115.2 & 0.4 & 4122.2 & 0.2 & 2.00 & C \\
\hline 30 & 1.6591 & 4116.9 & 0.2 & 4123.7 & 0.2 & 1.00 & PO \\
\hline 31 & 1.6598 & 4117.7 & 0.2 & 4124.8 & 0.2 & 1.00 & PR \\
\hline 32 & 1.6599 & 4118.2 & 0.7 & 4124.8 & 0.2 & 3.50 & PR \\
\hline 33 & 1.6636 & 4123.7 & 0.2 & 4130.7 & 0.1 & 2.00 & PO \\
\hline $33 a$ & 1.6689 & 4132.0 & 0.1 & 4138.7 & 0.8 & 0.13 & \\
\hline 34 & 1.6703 & 4134.2 & 0.9 & 4141.0 & 1.1 & 0.82 & C \\
\hline 35 & 1.6714 & 4135.9 & 0.9 & 4142.7 & $\cdot 1.4$ & 0.64 & C \\
\hline 36 & 1.6732 & 4138.7 & 0.8 & 4145.5 & 0.4 & 2.00 & C \\
\hline 37 & 1.6747 & 4141.0 & 1.1 & 4147.7 & 0.5 & 2.20 & C \\
\hline 38 & 1.6775 & 4145.5 & 0.4 & 4152.1 & 0.1 & 4.00 & PO \\
\hline 39 & 1.6820 & 4152.1 & 0.1 & 4159.3 & 0.2 & 0.50 & PR \\
\hline
\end{tabular}


Table I (Continued)

\begin{tabular}{|c|c|c|c|c|c|c|c|}
\hline \multirow[b]{2}{*}{$\begin{array}{l}\text { System } \\
\text { number }\end{array}$} & \multirow[b]{2}{*}{$z$} & \multicolumn{2}{|c|}{$\mathrm{C}$ IV $\lambda 1548.20$} & \multicolumn{2}{|c|}{$\mathrm{C}$ IV $\lambda 1550.77$} & \multirow[b]{2}{*}{$\mathrm{R}(\mathrm{EW})$} & \multirow[b]{2}{*}{$\begin{array}{l}\text { Credibility } \\
\text { rating }\end{array}$} \\
\hline & & $\lambda(\AA)$ & $\begin{array}{l}\text { Equivalent } \\
\text { width }(\AA)\end{array}$ & $\lambda(\AA)$ & $\begin{array}{l}\text { Equivalent } \\
\text { width }(\AA)\end{array}$ & & \\
\hline 40 & 1.6907 & 4165.6 & 0.3 & 4172.8 & 0.1 & 3.00 & PR \\
\hline 41 & 1.7188 & 4209.3 & 0.2 & 4216.1 & 0.2 & 1.00 & PO \\
\hline 42 & 1.7240 & 4217.4 & 0.1 & 4224.1 & 0.1 & 1.00 & PO \\
\hline 43 & 1.7292 & 4225.3 & 0.1 & 4232.1 & 0.2 & 0.50 & PO \\
\hline 44 & 1.7455 & 4250.6 & 0.1 & 4257.6 & 0.1 & 1.00 & PO \\
\hline 45 & 1.7533 & 4262.6 & 0.1 & 4269.7 & 0.2 & 0.50 & PO \\
\hline
\end{tabular}

redshift systems thus found are given in their Table 3. A check on these systems showed that the discrepancy allowed for the separation was more accurately $0.33 \AA$. To clarify the constraints imposed on the ratio $\mathrm{W}(\lambda 1548) / \mathrm{W}(\lambda 1551)$, we searched for all such pairs of lines whose separation would be the same as that of redshifted $C_{\text {IV }}$ lines, within $\pm 0.33 \AA$. A total of 52 such pairs were found and are shown in Table I. The forty-five pairs of BSBC are labelled 1 to 45 . The seven pairs rejected by BSBC are indicated by 3a, 4a, 6a, 9a, 12a, 13a, and 33a. The ratio of the equivalent widths,

$$
R(\mathrm{EW})=\frac{\mathrm{W}(\lambda 1548)}{\mathrm{W}(\lambda 1551)}
$$

is shown in column 7. BSBC have rated the credibility of the systems as follows: C-Certain, PR-Probable, and PO-Possible. These ratings are shown in the last column of Table I. An inspection of the $R(\mathrm{EW})$ values shows that considerable latitude was allowed. The accepted systems have $R(\mathrm{EW})$ lying between 0.11 to 4.0. As $R(\mathrm{EW})=0.11$ has been considered acceptable, very likely $R(\mathrm{EW})=5$ or 6 would have been accepted, had such a value occurred in the actual data. There are twelve cases with $R(\mathrm{EW})<1$ and eight cases with $R(\mathrm{EW})>2$.

BSBC recognized that many systems could arise by chance, and made a statistical assessment of the overall significance of the identifications. Earlier Varshni (1974b, c, 1975b) had emphasized the importance of taking into account the density distribution of lines for determining chance coincidence "redshifts". Since, in the case of PKS 0237-23, the distribution of lines in wavelength is not uniform, BSBC divided the line list into six $100 \AA$ regions and calculated the expected number of chance $C$ IV doublets in each region by the following formula

$$
E(C \text { IV })=\frac{N(N-1)}{L} \times 2 \Delta \lambda \times f,
$$

where $N$ is the number of lines in the region, $L$ is the extent of the region, $\Delta \lambda$ is the required accuracy of the separation, and $f$ is the probability of fulfilling the 
relative equivalent-width criterion by chance. The quantity $f$ was arbitrarily given a value 0.75 by BSBC. We have noted earlier that in the spectrum of PKS 0237-23 there are 52 systems, of which, BSBC have accepted 45 . It would be more logical to assume $f=45 / 52=0.87$. If the results of BSBC are taken at face value, these indicate that in two wavelength intervals, namely, $\lambda \lambda 4000-4100$ and $\lambda \lambda 4100-4200$, the number of observed systems is much larger than that expected by chance. Taken together, in the two intervals, there are 28 observed systems, whereas $14.1 \pm 3.5$ are expected by chance.

\section{The Error in the Work of BSBC}

There is a basic error in BSBC's calculation of the number of chance C IV doublets. They have not taken into account the variations in the density distribution of lines in the absorption spectrum of PKS 0237-23 in a proper way. The separation between the two $C$ IV lines varies from $6.17 \AA$ at $z=1.40$, to $7.09 \AA$ at $z=2.76$. Thus, it is seen that at any given redshift only an interval of about $10 \AA$ of the spectrum is sampled. In Figure 1 we show a histogram of the number of lines in $10 \AA$ intervals as a function of the wavelength. It will be noticed that at several places there are large fluctuations in the density of lines. The density of lines plays a very important role in determining chance coin-

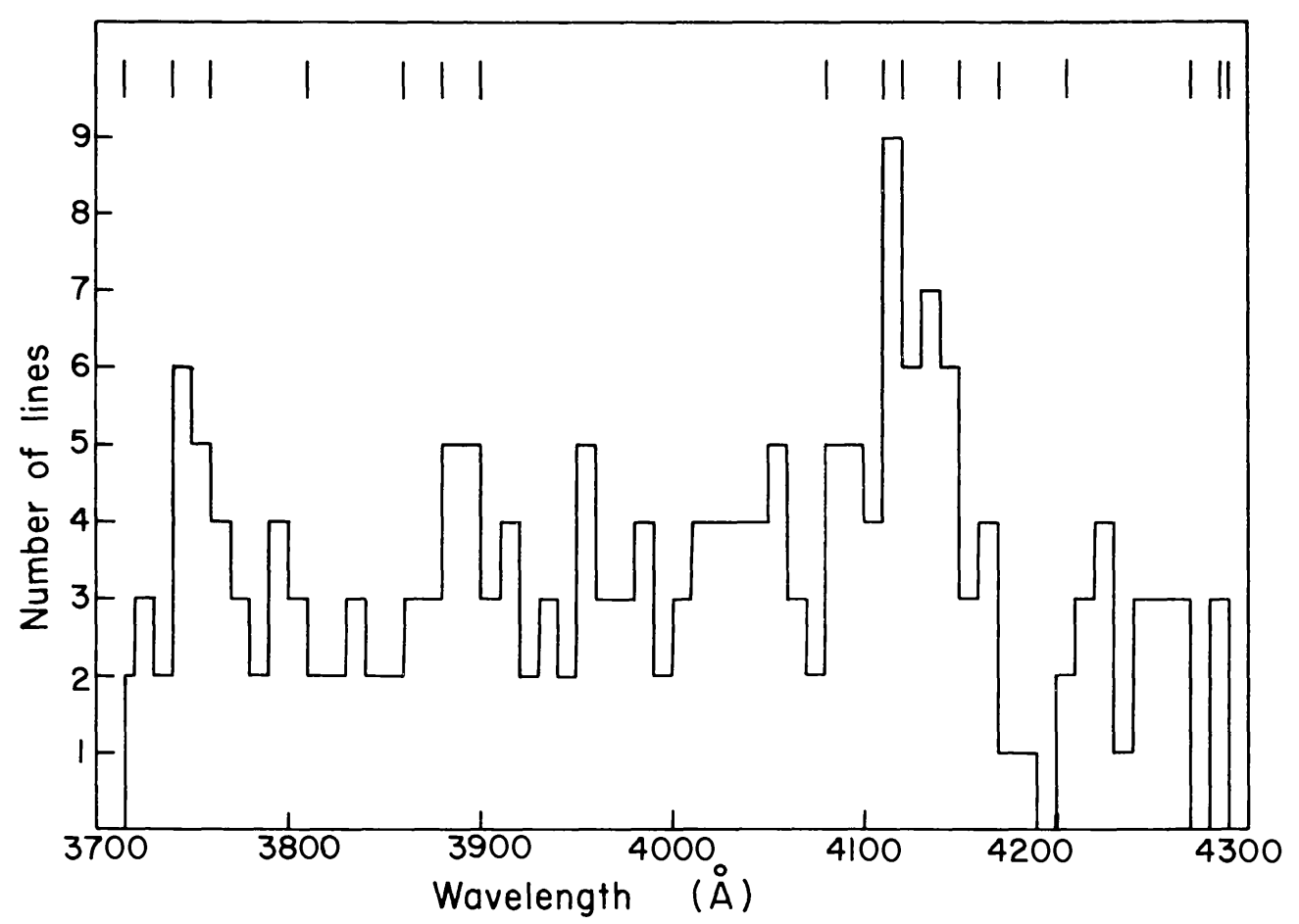

Fig. 1. Number of lines in $10 \AA$ intervals as a function of wavelength for PKS 0237-23. The data are due to BSBC. The vertical bars at the top indicate the positions of the "walls" used in generating ghost spectra. 
cidences (Russell and Bowen, 1929; Russell et al., 1944; Varshni, 1974b, c, 1975b). The fluctuations in the density of lines in the spectrum of PKS 0237-23 must be properly taken into account in any calculation of the number of chance coincidences.

\section{Chance Coincidences}

Let us calculate the chance coincidence probability of finding a $\mathrm{C}$ IV doublet at a certain redshift. Suppose there are $n$ observed lines in a wavelength interval $X$, and $x$ is the wavelength coincidence tolerance $(0.16 \AA$ in our case). Then the probability, $p_{1}$, of one of the observed lines falling within $\pm x$ of one of the $\mathrm{C}$ IV lines is

$$
p_{1}=2 n x / X
$$

Similarly,

$$
p_{2}=2(n-1) x /(X-2 x) .
$$

Thus, the probability of both the occurrences is

$$
P=\frac{4 n(n-1) x^{2}}{X(X-2 x)}
$$

For the mathematically-minded reader, we note that the BSBC procedure is equivalent to calculating $(1 / m)\left[\left(\sum_{i=1}^{m} a_{i}\right)^{2}-\sum_{i=1}^{m} a_{i}\right]$ as compared to the correct expression $\sum_{i=1}^{m} a_{i}\left(a_{i}-1\right)$, where $a_{i}$ represents a sequence of numbers. Only if the dispersion in the $a_{i}$ values is very small, the two expressions will be close to each other. If the dispersion in the $a_{i}$ 's is large, the former expression can be substantially smaller than the latter one.

The calculated values of $P$ in steps of $\Delta z=0.002$, as a function of $z$, are shown in Figure 2(a). These values were averaged over $\Delta z=0.02$ and the resulting histogram is shown in Figure 2(b), which may be compared with the results of BSBC, shown in Figure 2(c). The empty squares in Figure 2(c) represent the systems rejected by BSBC. It will be noticed that the chance-coincidence redshift distribution is very similar to that of the actual systems found for PKS 023723 . We conclude that the proposed systems are consistent with the hypothesis that these are just chance-coincidences without any physical significance.

To obtain the actual number of chance-coincidence redshift systems, two methods were used.

Method 1. A detailed examination of the data of BSBC and Figure 1 shows that the absorption spectrum of PKS 0237-23 can be conveniently divided into 15 intervals, to allow for the varying density of lines, with 'walls' at 3715, 3740, $3760,3810,3860,3880,3900,4080,4110,4120,4150,4170,4205,4270,4285$, and $4290 \AA$ A. Ten ghost spectra (Varshni, 1975b) were generated on a computer (IBM 


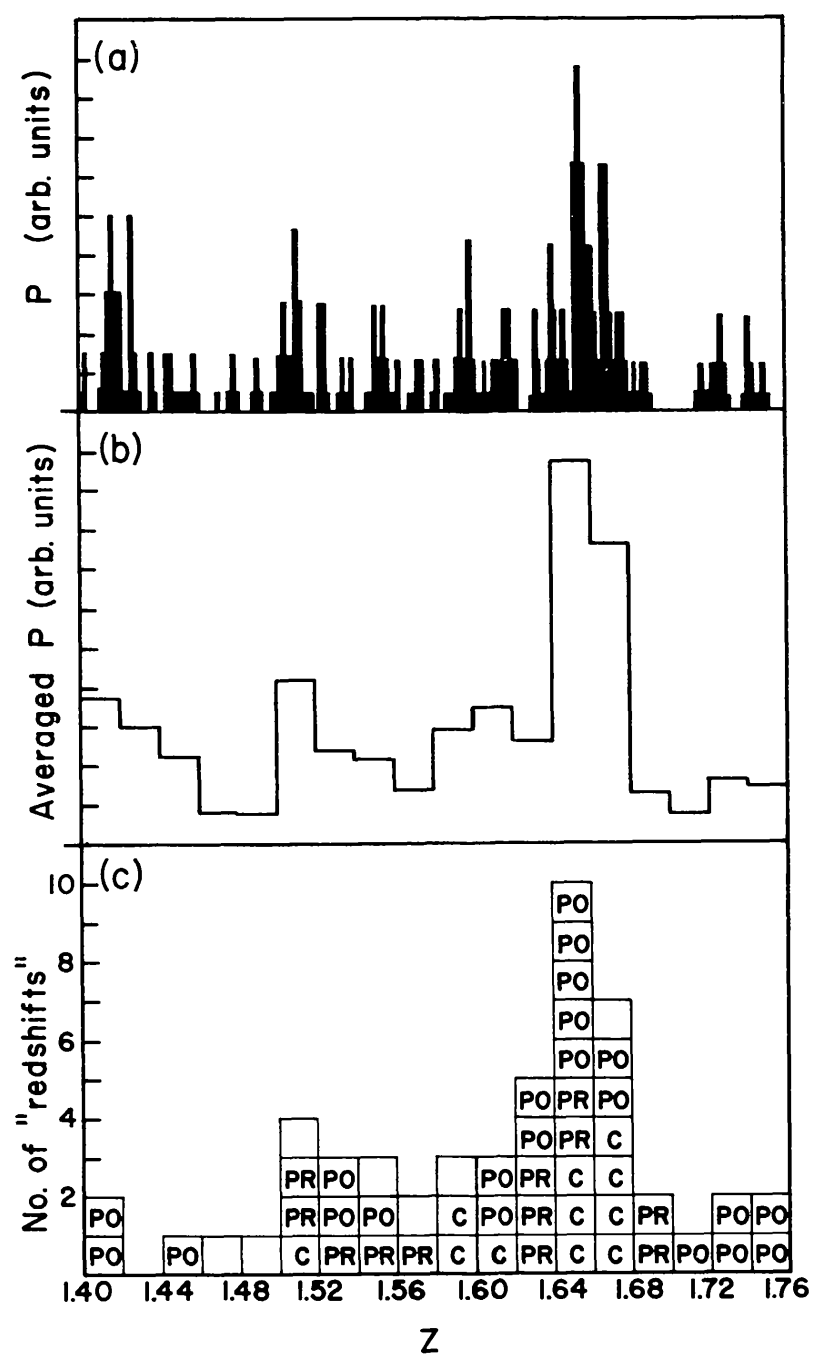

Fig. 2. (a) $P$ as a function of $z$, in steps of $\Delta z=0.002$. (b) Histogram showing averaged $P$ over $\Delta z=0.02$, as function of $z$. (c) Histogram showing the distribution of adsorption redshifts. The credibility rating of the 45 systems of BSBC is indicated by C, PR or PO.

360/65). The fifteen intervals were considered separately; inside an interval, the ghost wavelengths were generated by the formula

$$
\lambda_{i}(\text { ghost })=\lambda_{\min }+R_{i}\left(\lambda_{\max }-\lambda_{\min }\right),
$$

where $\lambda_{\min }$ and $\lambda_{\max }$ represent the lower and upper limits, respectively, of the interval, and $R_{i}$ is a uniformly distributed random number between zero and one (generated using the RANDU subroutine of IBM's Scientific Subroutine Package). The minimum separation between two lines in the spectrum reported by $\mathrm{BSBC}$ is $0.4 \AA$; it was constrained that the minimum separation between two wavelengths in a ghost spectrum will be the same. The equivalent width of the $i$ th observed line was assigned to $\lambda_{i}$ (ghost). Thus in any given interval, a ghost 
TABLE II

Number of C IV doublets in ghost spectra

\begin{tabular}{lll}
\hline $\begin{array}{l}\text { Ghost } \\
\text { spectrum } \\
\text { number }\end{array}$ & $\begin{array}{l}\text { Initiating } \\
\text { number } \\
\text { for RANDU }\end{array}$ & $\begin{array}{l}\text { Total } \\
\text { number of } \\
\text { C IV doublets }\end{array}$ \\
\hline 1 & 28471 & 53 \\
2 & 83123 & 47 \\
3 & 26687 & 45 \\
4 & 42003 & 48 \\
5 & 62893 & 50 \\
6 & 66981 & 49 \\
7 & 10737 & 47 \\
8 & 78241 & 50 \\
9 & 137 & 49 \\
10 & 8873 & 47 \\
& Average & 48.5 \\
& & \pm 2.1 \\
\hline
\end{tabular}

spectrum has the same number of lines of various equivalent widths as in the observed spectrum. Each ghost spectrum was analysed for 'redshifted' C IV doublets such that the separation between the lines is within $\pm 0.33 \AA$ of the correct separation. The initiating number used for RANDU subroutine for generating the ghost spectrum and the total number of $\mathrm{C}$ IV doublets found in each of the ten cases are shown in Table II. We have noted earlier that BSBC have not used any well-defined rule for $R(\mathrm{EW})$ for a system to be 'acceptable'. For the ghost spectra, we find that if we use the criterion that $R(\mathrm{EW})$ should lie between 0.11 and 5, approximately $85 \%$ of the systems are 'acceptable'. The average total number of chance $C$ IV doublets is $48.5 \pm 2.1$ and $\sim 85 \%$ of these are in the 'acceptable' category.

Method 2. The expression (1) (with $f=1$ ) was used to calculate the expected number of chance $C_{\text {IV }}$ doublets in each of the fifteen regions which are identified in Method 1. The total number of such doublets is found to be $48 \pm 6.9$. If we assume that $f=0.87$, the number of "acceptable" $C$ IV doublets comes out to be $41.8 \pm 6$. We may note here that this method does not take into account doublets formed such that the two lines lie in two separate, adjacent, intervals. However, the agreement of the result obtained by this method with that obtained from Method 1 indicates that the error arising because of this is small.

\section{Conclusions}

The results presented here conclusively show that the number of $\mathrm{C}$ IV redshift systems proposed by BSBC is insignificantly different from that that would be 
expected from chance coincidences. Consequently, these systems and their $z$ values are devoid of any physical significance. These $z$ values are merely a futile exercise in empty numerology.

The next, and more interesting, problem is what are these lines due to. We have succeeded in identifying a good many of the lines recorded by BSBC on the basis of the theory of quasars that we have proposed. We shall present these identifications and the arguments to support them in due course.

\section{Acknowledgement}

This work was supported in part by a research grant from the National Research Council of Canada.

\section{References}

Arp, H. C., Bolton, J. G. and Kinman, T.: 1967, Astrophys. J. 147, 841.

Boksenberg, A. and Sargent, W. L. W.: 1975, Astrophys. J. 198, 31.

Boroson, T., Sargent, W. L. W., Boksenberg, A. and Carswell, R. F.: 1978, Astrophys. J. $220,772$.

Burbidge, E. M., Lynds, C. R. and Stockton, A. N.: 1968, Astrophys. J. 152, 1077.

Greenstein, J. L., and Schmidt, M.: 1967, Astrophys. J. 148, L13.

Russell, H. N. and Bowen, I. S.: 1929, Astrophys. J. 69, 196.

Russell. H. N., Moore, C. E. and Weeks, D. W.: 1944, Trans. Am. Phil. Soc. 34, 111.

Varshni, Y. P.: 1973, Bull. Am. Phys. Soc. 18, 1384.

Varshni, Y. P.: 1974a, Bull. Am. Astron. Soc. 6, 213, 308.

Varshni, Y. P.: 1974b, Astrophys. J. 193, L5.

Varshni, Y. P.: 1974c, Bull. Am. Astron. Soc. 6, 449.

Varshni, Y. P.: 1975a, Astrophys. Space Sci. 37, L1.

Varshni, Y. P.: 1975b, Astrophys. J. 201, 547.

Varshni, Y. P.: 1977a, Astrophys. Space Sci. 46, 443.

Varshni, Y. P.: 1977b, J. Roy. Astron. Soc. Canada 71, 403.

Varshni, Y. P.: 1978, in S. Fujita (ed.), The Ta-You Wu Festschrift: Science of Matter, Gordon and Breach, New York, p. 285.

Varshni, Y. P.: 1979, Physics in Canada 35, 11.

Varshni, Y. P. and Lam, C. S.: 1976, Astrophys. Space Sci. 45, 87. 